\title{
K- NEAREST NEIGHBORS BASED AUTOMATIC MODULATION CLASSIFIER FOR NEXT GENERATION ADAPTIVE RADIO SYSTEMS
}

\author{
M. Venkata Subbarao ${ }^{1 *}$ and P. Samundiswary ${ }^{2}$ \\ ${ }^{1}$ Research Scholar, Department of EE \\ ${ }^{2}$ Assistant Professor, Department of EE \\ ${ }^{1,2}$ School of Engineering \& Technology, Pondicherry University, India \\ ${ }^{1}$ mandava.decs@gmail.com
}

\begin{abstract}
This paper presents a verity of K-Nearest Neighbors (KNN) classifiers along with different statistical features in order to classify MPSK and MQAM signals. Further, the performance of proposed KNN classifiers with different values of ' $K$ ' and distance functions are analyzed under non-ideal channel conditions. Finally, to prove the superiority of the proposed KNN classifiers the performance is compared with that of the literature approaches at various SNR values.
\end{abstract}

Keywords - Modulation Recognition, Distance Function, Nearest Neighbors, Majority Selection

\section{INTRODUCTION}

Security is the key issue in many communication applications especially for military applications [1]. In contrast to this, the authorities of boarder security may wish to monitor unlicensed transmitters for jamming their signals. The necessary action of doing so is to identify or recognize the modulation class of that intercepted signal. Such type of actions also arise in several other applications such as interference management, signal authorization, verification, and selection of appropriate demodulation techniques in electronic combat, threat analysis, and so on. Modulation Recognition (MR) is also useful to recognize the suspicious transmitter in the near geographical site and to generate jamming signals to stop communication between the suspicious users. On the other hand, AMC provides as an additional level of encryption to the data and preventing unknown receivers from recovering the information without any awareness of the modulation type [2]. In recent civilian applications, more number of modulation formats can be employed by a transmitter to manage the data rate, to reduce the individual bandwidths of every user, and to assure the integrity of the message. However, the group of modulation formats is known both to transmitter and receiver. The choice of the modulation format is adaptive and may not be known at the receiving end. Therefore, an AMC mechanism is required for the receiving end to recognize format of modulated signal and to select the proper demodulation approach in order to recover the original message. Therefore, MR becomes an essential part of future Adaptive Radio Systems (ARSs) [3].

Broadly MR approaches are classified in to joint Time-Frequency Analysis (TFA), Decision Theoretic (DTC) or Maximum Likelihood (ML), Wavelet Transform (WT), statistical and Feature Based (FB) or Pattern Recognition (PR) approaches [4-9]. Amplitude and frequency variations of the signal can be effectively tracked by TFA but it is unable to track the phase variations in the signal. Hence, TFA is capable in

Received: September 13, 2019

Reviewed: November 21, 2019

Accepted: December 6, 2019

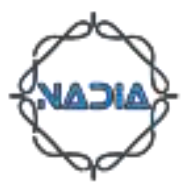


classification of MASK, MFSK AM and FM modulated signals but not of MQAM and M-ary PSK signals [10-11].

In DTC approaches [12-15], a prior knowledge about signal and channel characteristics is necessary to get optimum performance which is impractical. These algorithms are computationally complex, and these are sensitive to phase, frequency offsets and synchronization errors. In order to reduce the computations and to avoid the prior information of the signal characteristics, a new approach namely wavelet transform method is introduced for MR [16-18]. It has the capability of extracting the transient information about the variations in signal amplitude, phase, and frequency. The extracted transient information is capable of classifying the signal through simple methods.

A statistical signal method extracts the modulation type by considering different nonlinearity properties such as higher order correlation, moments and cumulants in complex envelope of the signal. These HOS and cyclostationary statistics of signals are further used for classification [19-22]. Statistical approaches are easy to implement and require very less time for recognition of accurate modulation type. However, the selection of right feature set is required in order to achieve improved performance by the statistical approaches. The FB approaches eliminates the need of prior information about the signal and channel. So these approaches are practically realizable and are able to work under different noisy conditions [23-26]. Recently, PR approaches such as Neural Network (NN), Back Propagation Neural Network (BPNN) in order to achieve better classification accuracy than Kolmogorov Smirnov (KS) and HoS approaches [27], [28].

The organization of the article is as follows: the feature extraction and feature selection is discussed in Section 2. KNN algorithm for MR is presented in Section 3. Simulation results of KNN based MR algorithm in non-ideal channel conditions is discussed in Section 4. Finally, section 5 depicts the critical conjectures of the paper.

\section{SYSTEM MODEL}

The received signal at receiver $r(n)$ is given by

$$
r(n)=m(n)+f(n)
$$

where $m(n)$ is a signal which is transmitted and $f(n)$ is fading noise added in the channel.

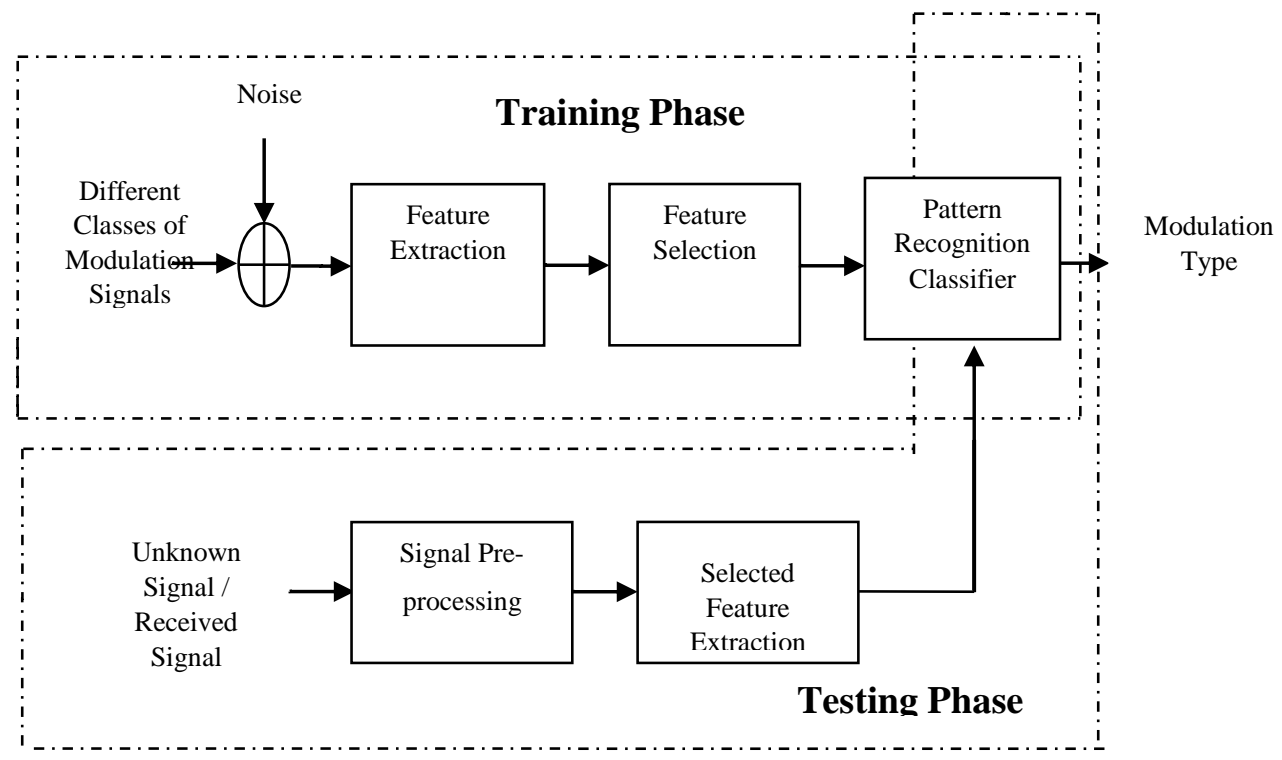

Fig. 1 Framework of Proposed Approach 
The received signal $r(n)$ is given by [3]

$$
r(n)=A e^{i\left(2 \pi n T f_{0}+\theta_{n}\right)} \sum_{k=-\infty}^{m} m(k) h((n-k+\epsilon) T)
$$

here $m(k)$ is input binary stream, $h($.$) is channel effects, \theta_{n}$ is phase jitter, ${ }^{\boldsymbol{\epsilon}}$ is time shifts occurred in channe, $A$ is the amplitude and $T$ is symbol time.

The diagrammatic procedure of proposed MR scheme is shown in Fig. 1. Initially a set of 39 statistical features are extracted from each modulation class signal which includes cumulants, moments and ratios. Thereafter, to reduce the training time and classification time, a set of 11 noise robust features are chosen using rough set theory investigations. For classification, proposed KNN classifiers are trained with the selected features of all modulation classes. In the testing phase, the selected features of the signal are extracted and passed through a trained PRC to recognize the modulation format of an unknown modulated signal.

The moments of the signal depend on the order of the statistical features, and they are given by [2]

$$
M_{p q}=E\left[r(n)^{p-q} r^{*}(n)^{q}\right]
$$

Here $p, q$ are the integers, $r(n)$ is the received signal, and $r^{*}(n)$ is the complex conjugate of the received signal.

Multi-order moments $M_{20}, M_{21}, M_{22}, M_{40}, M_{41}, M_{42}, M_{41}, M_{60}, M_{61}, M_{62}, M_{61}, M_{80} \quad$ and $M_{94}$ are calculated from the equation (3) to derive the multi-order cumulants. The cumulants of a signal are a set of statistical quantities that provide an alternative to the moments of the distribution and these are derived from moments [3].

A set of 11 noise robust features $C_{20}, C_{21}, C_{40}, C_{41}, C_{42}, C_{60}, C_{61}, C_{62}, C_{61}, C_{80}$, and $C_{84}$ are selected for training the proposed PRC's. A set selected features are extracted from an unknown received signal after signal pre-processing. The extracted features are further passed through the trained PRC's for modulation reorganization.

\section{KNN BASED PRC FOR AMC}

The KNN is a supervised learning classifier and it is capable of classifying multi-class objects [29]. To classify a signal, KNN algorithm measures similarity index based on distance with all available classes of signals, and then the signal is assigned to a modulation class through a majority vote among its K-nearest neighbors. Based on size of data set, the value of $\mathrm{K}$ is chosen and it decides the performance of the classifier. In KNN approach $\mathrm{K}$ value and distance function affects the performance of the classifier. In general, higher $\mathrm{K}$ value minimizes the impact of the noise on the classification accuracy, but makes margins among classes with less distinct. The algorithmic approach for KNN classifier is shown in Fig. 2.

\footnotetext{
Inputs:

1. Set of ' $N$ ' reference modulated signals from each class of modulation under various noise conditions with a set of extracted features sets $\boldsymbol{F}_{R}$

2. Extracted set of feature $\boldsymbol{F}_{u}$ of an unknown signal is to be recognized.

3. Pre-defined $\mathrm{K}$ value and distance function.

Output: Specific Modulation Class
}

Step 1: Determine the distance between $\boldsymbol{F}_{u \text { to }}$ all reference feature sets $\boldsymbol{F}_{\boldsymbol{R}}$ is calculated using predefined distance function.

Step 2: Keeps the distance values $\mathrm{D}\left(\boldsymbol{F}_{u}, \boldsymbol{F}_{\boldsymbol{R}}\right)$ in descending order 
Step 3: Stores the modulation class of each distance value in D.

Step 4: Select the first $\mathrm{K}$ distance values from $\mathrm{D}$.

Step 5: Extract the modulation classes of first $\mathrm{K}$ distance values.

Step 6: Find the majority class among the first $\mathrm{K}$ modulation classes and returned as a classification decision.

Fig. 2 Algorithmic approach for proposed KNN classifier

Based on the K value and distance functions, the KNN's are subcategorized into Fine KNN, Medium KNN, Coarse KNN, Cosine KNN, Cubic KNN, and Weighted KNN. The characteristics of each KNN are shown in Table I [24]. The performance of proposed KNN classifier is tested with various values of $\mathrm{K}$ such as 1,10 and 100 , and with different distance functions such as Euclidean, cosine and cubic distances. In weighted $\mathrm{KNN}$, the decision is taken by calculating the weighting function from the Euclidean distance value.

Table I. KNN Classifiers

\begin{tabular}{|c|c|c|c|}
\hline Classifier Type & $\mathrm{K}$ & Distance Function & Distance $(\mathrm{d})$ \\
\hline Fine KNN & 1 & Euclidean Distance & $\sqrt{\sum_{\mathrm{i}=1}^{n}\left(p_{\mathrm{i}}-q_{\mathrm{i}}\right)^{2}}$ \\
\hline Medium KNN & 10 & Euclidean Distance & $\sqrt{\sum_{\mathrm{i}=1}^{n}\left(p_{\mathrm{i}}-q_{\mathrm{i}}\right)^{2}}$ \\
\hline Coarse KNN & 100 & Euclidean Distance & $\sqrt{\sum_{i=1}^{n}\left(p_{\mathrm{i}}-q_{\mathrm{i}}\right)^{2}}$ \\
\hline Cosine KNN & 10 & Cosine Distance & $\frac{\bar{P} . \bar{Q}}{|\vec{P} \| \vec{Q}|}$ \\
\hline Cubic KNN & 10 & Cubic Distance & $\left(\sum_{\mathrm{i}=1}^{m}\left|p_{\mathrm{i}}-q_{\mathrm{i}}\right|^{\mid{ }^{2}}\right)^{1 / a}$ \\
\hline Weighted KNN & 10 & $\begin{array}{c}\text { Distance Weighting \& } \\
\text { Euclidean Distance }\end{array}$ & $w=1 / d^{2}$ \\
\hline
\end{tabular}

\section{SIMULATION RESULTS AND DISCUSSIONS}

The performance analysis of proposed KNN classifiers for AMC is carried out under non-ideal channel conditions. MPSK (with $\mathrm{M}=2$, 4 and 8), 4QAM, 16QAM, and 64QAM signals are considered for the simulation to validate the efficiency of proposed PRC. Several multipath fading conditions are considered by varying the number of paths, frequency selective fading along with AWGN conditions for different values of SNR from 0 to $20 \mathrm{~dB}$.

From the experimental tests, 1000 copies of each modulation class are considered under varying noise conditions. For each copy of the modulated signal, a set of 11 selective statistical features are extracted for training and testing. The performance measures are carried out with $90 \%$ percentage of training and $10 \%$ testing. The performance metrics of the proposed PRC are classification accuracy or true positive rate and false negative rates. Table II represents the confusion matrix for different KNN classifiers using multi-order cumulants at different SNRs. Diagonal elements in the 
confusion matrix denotes the true classification rates and off diagonal elements represent false negative rates.

The classification performance of Fine KNN and Medium KNN with 90\% training is shown in Fig. 3 and it is clear that Fine KNN classifier is unable to classify the 8PSK, 16QAM and 64QAM modulation classes at lower SNR values. Therefore, the average accuracy of Fine KNN and Medium KNN at $0 \mathrm{~dB}$ is $79.4 \%$ and $78.9 \%$ respectively.
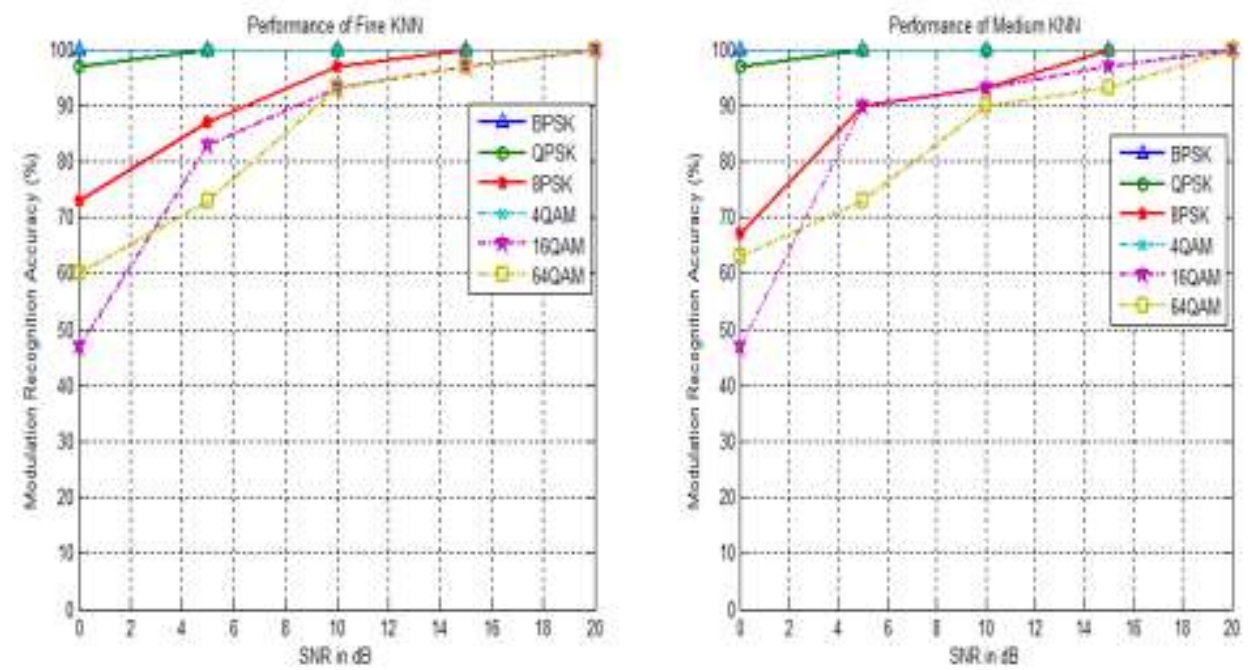

Fig. 3 Performance of Fine and Medium KNN Classifiers
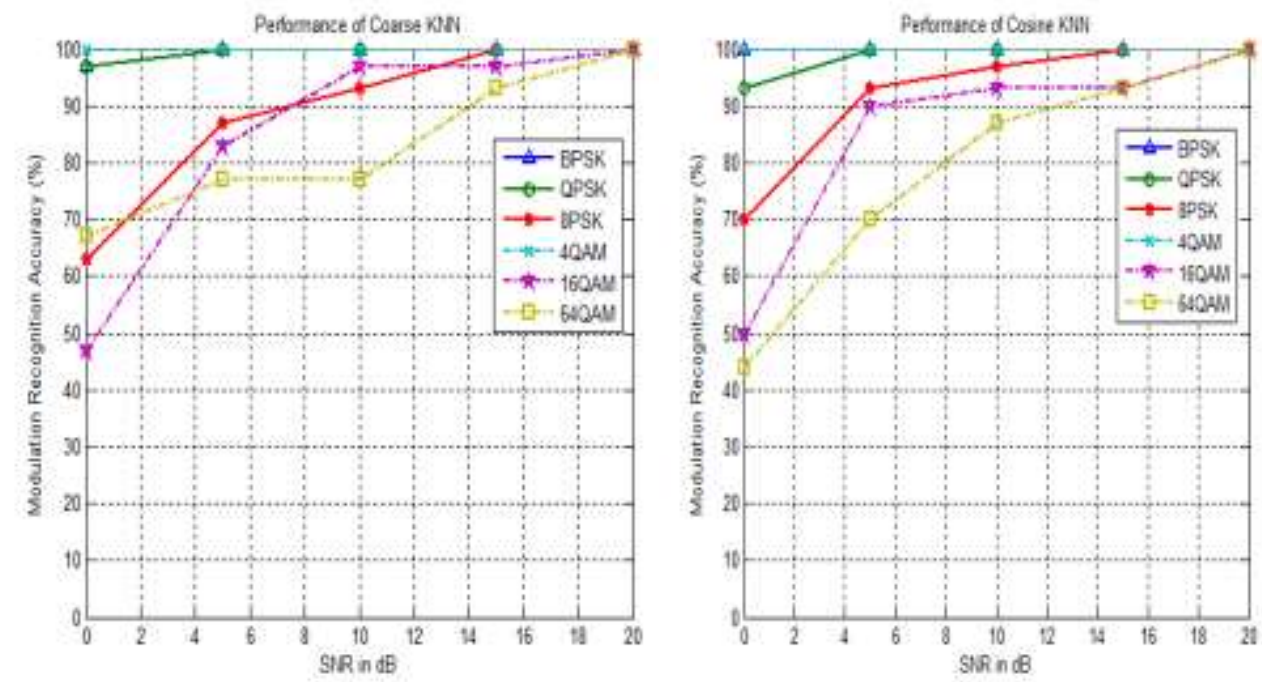

Fig. 4 Performance of Coarse and Cosine KNN Classifiers

The classification performance of Coarse KNN and Cosine KNN is shown in Fig. 4. Coarse KNN classifier provides fewer distinctions than a Fine KNN and Medium KNN. The average classification accuracy of Coarse KNN and Cosine KNN at $0 \mathrm{~dB}$ are $78.3 \%$ and $76.1 \%$ respectively.

The classification performance of Cubic KNN and Weighted KNN is shown in Fig. 5. Cosine KNN measures cosine distance to make distinctions between the classes, where as Cubic KNN uses cubic distance. The classification accuracy of Cubic and weighted KNN's at $0 \mathrm{~dB}$ are $80 \%$ and $77.2 \%$ respectively. 
Table II. Confusion matrix of proposed KNN classifiers with 90\% Training

\begin{tabular}{|c|c|c|c|c|c|c|c|c|c|c|c|c|c|c|c|c|c|c|c|c|c|c|c|c|c|c|c|c|c|c|c|}
\hline \multirow{3}{*}{ Classifier } & \multirow{3}{*}{$\begin{array}{c}\begin{array}{c}\text { True } \\
\text { Class }\end{array} \\
\text { BPSK }\end{array}$} & \multicolumn{30}{|c|}{ SNR } \\
\hline & & \multicolumn{6}{|c|}{$0 \mathrm{~dB}$} & \multicolumn{6}{|c|}{$5 \mathrm{~dB}$} & \multicolumn{6}{|c|}{$10 \mathrm{~dB}$} & \multicolumn{6}{|c|}{$15 \mathrm{~dB}$} & \multicolumn{6}{|c|}{$20 \mathrm{~dB}$} \\
\hline & & 100 & $\mathbf{0}$ & $\mathbf{0}$ & 0 & $\mathbf{0}$ & $\mathbf{0}$ & 100 & o & 0 & 0 & 0 & O & 100 & 0 & $\mathbf{0}$ & 0 & 0 & O & 10 & $\begin{array}{lll}0 & 0\end{array}$ & 0 & 0 & $\mathbf{0}$ & 0 & 100 & O & 0 & 0 & 0 & 0 \\
\hline \multirow{5}{*}{$\begin{array}{l}\text { Fine } \\
\text { KNN }\end{array}$} & QPSK & $\mathbf{0}$ & 97 & $\mathbf{0}$ & $\mathbf{0}$ & $\mathbf{0}$ & 3 & $\mathbf{0}$ & 100 & $\mathbf{0}$ & 0 & $\mathbf{0}$ & $\mathbf{0}$ & $\mathbf{0}$ & 100 & $\mathbf{0}$ & $\mathbf{0}$ & $\mathbf{0}$ & $\mathbf{0}$ & $\mathbf{0}$ & 100 & $\mathbf{0}$ & $\mathbf{0}$ & $\mathbf{0}$ & 0 & $\mathbf{0}$ & 100 & $\mathbf{0}$ & $\mathbf{0}$ & 0 & $\mathbf{0}$ \\
\hline & 8PSK & $\mathbf{0}$ & $\mathbf{0}$ & 73 & $\mathbf{0}$ & 14 & 13 & $\mathbf{0}$ & $\mathbf{0}$ & 87 & $\mathbf{0}$ & 13 & $\mathbf{0}$ & 0 & $\mathbf{0}$ & 97 & $\mathbf{0}$ & 3 & 0 & $\mathbf{0}$ & 0 & 100 & $\mathbf{0}$ & $\mathbf{0}$ & $\mathbf{0}$ & $\mathbf{0}$ & 0 & 100 & 0 & $\mathbf{0}$ & $\mathbf{0}$ \\
\hline & 4QAM & 0 & $\mathbf{0}$ & $\mathbf{0}$ & 100 & 0 & $\mathbf{0}$ & $\mathbf{0}$ & $\mathbf{0}$ & $\mathbf{0}$ & 100 & $\mathbf{0}$ & $\mathbf{0}$ & $\mathbf{0}$ & $\mathbf{0}$ & $\mathbf{0}$ & 100 & $\mathbf{0}$ & 0 & $\mathbf{0}$ & 0 & $\mathbf{0}$ & 100 & $\mathbf{0}$ & 0 & 0 & 0 & 0 & 100 & $\mathbf{0}$ & 0 \\
\hline & 16QAM & o & $\mathbf{0}$ & 23 & 0 & 47 & 30 & o & o & 4 & o & 83 & 13 & 0 & 0 & $\mathbf{0}$ & 0 & 93 & 7 & $\mathbf{0}$ & O & 3 & O & 97 & 0 & o & o & 0 & o & 100 & $\mathbf{0}$ \\
\hline & 64QAM & 0 & 3 & 3 & 0 & 34 & 60 & 0 & 3 & $\mathbf{0}$ & 0 & 24 & 73 & 0 & 0 & $\mathbf{0}$ & 0 & 7 & 93 & $\mathbf{0}$ & 0 & 0 & 0 & 3 & 97 & 0 & o & 0 & o & 0 & 100 \\
\hline \multirow{6}{*}{$\begin{array}{l}\text { Medium } \\
\text { KNN }\end{array}$} & BPSK & 100 & $\mathbf{0}$ & 0 & 0 & $\mathbf{0}$ & $\mathbf{0}$ & 100 & o & 0 & 0 & 0 & $\mathbf{0}$ & 100 & 0 & $\mathbf{0}$ & 0 & $\mathbf{0}$ & 0 & 10 & $\begin{array}{lll}0 & 0\end{array}$ & 0 & 0 & $\mathbf{0}$ & 0 & 100 & o & o & 0 & 0 & 0 \\
\hline & QPSK & o & 97 & $\mathbf{0}$ & 0 & 3 & 0 & o & 100 & 0 & o & $\mathbf{0}$ & 0 & 0 & 100 & 0 & o & 0 & o & $\mathbf{0}$ & 100 & o & o & $\mathbf{0}$ & 0 & O & 100 & 0 & o & o & o \\
\hline & 8PSK & $\mathbf{0}$ & $\mathbf{0}$ & 67 & $\mathbf{0}$ & 27 & 6 & $\mathbf{0}$ & $\mathbf{0}$ & 90 & $\mathbf{0}$ & 10 & $\mathbf{0}$ & $\mathbf{0}$ & $\mathbf{0}$ & 93 & $\mathbf{0}$ & 7 & 0 & $\mathbf{0}$ & 0 & 100 & $\mathbf{0}$ & $\mathbf{0}$ & $\mathbf{0}$ & $\mathbf{0}$ & $\mathbf{0}$ & 100 & $\mathbf{0}$ & $\mathbf{0}$ & $\mathbf{0}$ \\
\hline & 4QAM & $\mathbf{0}$ & $\mathbf{0}$ & $\mathbf{0}$ & 100 & 0 & $\mathbf{0}$ & $\mathbf{0}$ & 0 & $\mathbf{0}$ & 100 & $\mathbf{0}$ & $\mathbf{0}$ & 0 & $\mathbf{0}$ & $\mathbf{0}$ & 100 & $\mathbf{0}$ & $\mathbf{0}$ & $\mathbf{0}$ & 0 & 0 & 100 & $\mathbf{0}$ & 0 & $\mathbf{0}$ & 0 & 0 & 100 & 0 & 0 \\
\hline & 16QAM & 0 & $\mathbf{0}$ & 23 & 0 & 47 & 30 & 0 & 0 & 3 & $\mathbf{0}$ & 90 & 7 & 0 & 0 & $\mathbf{0}$ & o & 93 & 7 & $\mathbf{0}$ & 0 & 0 & $\mathbf{0}$ & 97 & 3 & 0 & 0 & 0 & 0 & 100 & 0 \\
\hline & 64QAM & 0 & 3 & 4 & 0 & 30 & 63 & 0 & 3 & $\mathbf{0}$ & 0 & 24 & 73 & 0 & 0 & $\mathbf{0}$ & o & 10 & 90 & $\mathbf{0}$ & 0 & 0 & 0 & 7 & 93 & 0 & 0 & 0 & 0 & 0 & 100 \\
\hline \multirow{6}{*}{$\begin{array}{l}\text { Coarse } \\
\text { KNN }\end{array}$} & BPSK & 97 & 3 & $\mathbf{0}$ & 0 & $\mathbf{0}$ & o & 100 & 0 & 0 & 0 & $\mathbf{0}$ & $\mathbf{0}$ & 97 & 3 & $\mathbf{0}$ & $\mathbf{0}$ & $\mathbf{0}$ & 0 & $\begin{array}{c}\mathbf{1 0} \\
\mathbf{0}\end{array}$ & 0 & 0 & 0 & $\mathbf{0}$ & 0 & 100 & 0 & 0 & 0 & 0 & 0 \\
\hline & QPSK & 0 & 97 & $\mathbf{0}$ & 0 & 3 & 0 & 0 & 100 & 0 & o & $\mathbf{0}$ & $\mathbf{0}$ & 0 & 100 & $\mathbf{0}$ & o & 0 & 0 & 0 & 100 & 0 & o & 0 & 0 & o & 100 & 0 & 0 & o & 0 \\
\hline & 8PSK & 0 & $\mathbf{0}$ & 63 & o & 30 & 7 & o & o & 87 & o & 13 & $\mathbf{0}$ & 0 & o & 93 & $\mathbf{0}$ & 7 & o & $\mathbf{0}$ & o & 100 & 0 & $\mathbf{0}$ & 0 & o & o & 100 & 0 & 0 & 0 \\
\hline & 4QAM & 0 & $\mathbf{0}$ & 0 & 100 & $\mathbf{0}$ & $\mathbf{0}$ & 0 & 0 & 0 & 100 & 0 & $\mathbf{0}$ & 0 & 0 & $\mathbf{0}$ & 100 & $\mathbf{0}$ & 0 & $\mathbf{0}$ & 0 & 0 & 100 & $\mathbf{0}$ & 0 & 0 & 0 & 0 & 100 & 0 & 0 \\
\hline & 16QAM & 0 & $\mathbf{0}$ & 26 & 0 & 47 & 27 & 0 & 0 & 3 & 0 & 83 & 14 & 0 & 0 & $\mathbf{0}$ & o & 97 & 3 & $\mathbf{0}$ & 0 & 0 & $\mathbf{0}$ & 97 & 3 & 0 & 0 & 0 & 0 & 100 & 0 \\
\hline & 64QAM & 0 & 6 & $\mathbf{0}$ & o & 27 & 67 & 0 & o & 0 & o & 23 & 77 & o & o & $\mathbf{0}$ & $\mathbf{0}$ & 23 & 77 & $\mathbf{0}$ & o & o & o & 7 & 93 & o & 0 & o & 0 & O & 100 \\
\hline \multirow{6}{*}{$\begin{array}{l}\text { Cosine } \\
\text { KNN }\end{array}$} & BPSK & 100 & $\mathbf{0}$ & $\mathbf{0}$ & 0 & $\mathbf{0}$ & o & 100 & 0 & 0 & 0 & $\mathbf{0}$ & $\mathbf{0}$ & 100 & 0 & $\mathbf{0}$ & $\mathbf{0}$ & $\mathbf{0}$ & 0 & $\begin{array}{c}\mathbf{1 0} \\
\mathbf{0}\end{array}$ & 0 & 0 & 0 & $\mathbf{0}$ & 0 & 100 & 0 & 0 & 0 & 0 & 0 \\
\hline & QPSK & 0 & 93 & $\mathbf{0}$ & 0 & $\mathbf{0}$ & 7 & 0 & 100 & 0 & 0 & $\mathbf{0}$ & $\mathbf{0}$ & 0 & 100 & $\mathbf{0}$ & $\mathbf{0}$ & $\mathbf{0}$ & 0 & $\mathbf{0}$ & 100 & 0 & 0 & $\mathbf{0}$ & 0 & 0 & 100 & 0 & 0 & 0 & 0 \\
\hline & 8PSK & 0 & $\mathbf{0}$ & 70 & 0 & 23 & 7 & 0 & 0 & 93 & 0 & 7 & $\mathbf{0}$ & 0 & 0 & 97 & 0 & 3 & 0 & $\mathbf{0}$ & 0 & 100 & 0 & $\mathbf{0}$ & 0 & 0 & 0 & 100 & 0 & 0 & 0 \\
\hline & 4QAM & 0 & $\mathbf{0}$ & 0 & 100 & $\mathbf{0}$ & $\mathbf{0}$ & o & 0 & 0 & 100 & 0 & $\mathbf{0}$ & o & 0 & $\mathbf{0}$ & 100 & $\mathbf{0}$ & 0 & $\mathbf{0}$ & 0 & 0 & 100 & $\mathbf{0}$ & 0 & o & 0 & 0 & 100 & 0 & 0 \\
\hline & 16QAM & 0 & $\mathbf{0}$ & 27 & 0 & 50 & 23 & 0 & 0 & 3 & 0 & 90 & 7 & 0 & $\mathbf{0}$ & 3 & o & 93 & 4 & $\mathbf{0}$ & 0 & 7 & $\mathbf{0}$ & 90 & 3 & 0 & 0 & 0 & 0 & 100 & 0 \\
\hline & 64QAM & 0 & 3 & $\mathbf{0}$ & 0 & 53 & 44 & 0 & 10 & 0 & 0 & 20 & 70 & 0 & 3 & $\mathbf{0}$ & o & 10 & 87 & $\mathbf{0}$ & 0 & 3 & 0 & 4 & 93 & 0 & 0 & 0 & 0 & 0 & 100 \\
\hline \multirow{6}{*}{$\begin{array}{l}\text { Cubic } \\
\text { KNN }\end{array}$} & BPSK & 97 & 3 & $\mathbf{0}$ & 0 & $\mathbf{0}$ & $\mathbf{0}$ & 100 & 0 & o & 0 & $\mathbf{0}$ & $\mathbf{0}$ & 100 & 0 & $\mathbf{0}$ & $\mathbf{0}$ & $\mathbf{0}$ & 0 & $\begin{array}{c}10 \\
0\end{array}$ & $\mathbf{0}$ & 0 & 0 & $\mathbf{0}$ & 0 & 100 & 0 & 0 & 0 & 0 & 0 \\
\hline & QPSK & 0 & $\begin{array}{c}\mathbf{1 0} \\
\mathbf{0}\end{array}$ & $\mathbf{0}$ & 0 & $\mathbf{0}$ & $\mathbf{0}$ & 0 & 100 & 0 & 0 & $\mathbf{0}$ & $\mathbf{0}$ & 0 & 100 & 0 & $\mathbf{0}$ & $\mathbf{0}$ & 0 & $\mathbf{0}$ & 100 & 0 & 0 & $\mathbf{0}$ & 0 & 0 & 100 & 0 & 0 & 0 & 0 \\
\hline & $8 \mathrm{PSK}$ & 0 & $\mathbf{0}$ & 67 & 0 & 30 & 3 & 0 & o & 90 & o & 10 & $\mathbf{0}$ & 0 & 0 & 93 & o & 7 & $\mathbf{0}$ & $\mathbf{0}$ & 0 & 100 & 0 & 0 & 0 & o & o & 100 & 0 & 0 & 0 \\
\hline & 4QAM & 0 & $\mathbf{0}$ & $\mathbf{0}$ & 100 & $\mathbf{0}$ & o & 0 & 0 & 0 & 100 & $\mathbf{0}$ & $\mathbf{0}$ & 0 & 0 & $\mathbf{0}$ & 100 & $\mathbf{0}$ & $\mathbf{0}$ & $\mathbf{0}$ & 0 & 0 & 100 & $\mathbf{0}$ & 0 & 0 & 0 & 0 & 100 & 0 & 0 \\
\hline & 16QAM & 0 & $\mathbf{0}$ & 20 & 0 & 50 & 30 & 0 & 0 & 3 & 0 & 90 & 7 & 0 & 0 & $\mathbf{0}$ & o & 93 & 7 & $\mathbf{0}$ & 0 & 0 & $\mathbf{0}$ & 97 & 3 & 0 & 0 & 0 & 0 & 100 & 0 \\
\hline & 64QAM & 0 & 3 & 3 & 0 & 27 & 67 & 0 & 3 & 0 & 0 & 17 & 80 & 0 & 0 & $\mathbf{0}$ & o & 10 & 90 & $\mathbf{0}$ & 0 & 0 & $\mathbf{0}$ & 7 & 93 & 0 & 0 & 0 & 0 & 0 & 100 \\
\hline \multirow{6}{*}{$\begin{array}{c}\text { Weighted } \\
\text { KNN }\end{array}$} & BPSK & 97 & 3 & $\mathbf{0}$ & 0 & $\mathbf{0}$ & $\mathbf{0}$ & 100 & 0 & 0 & 0 & $\mathbf{0}$ & $\mathbf{0}$ & 100 & 0 & $\mathbf{0}$ & $\mathbf{0}$ & $\mathbf{0}$ & 0 & $\begin{array}{c}\mathbf{1 0} \\
\mathbf{0}\end{array}$ & 0 & 0 & 0 & $\mathbf{0}$ & 0 & 100 & 0 & 0 & 0 & 0 & 0 \\
\hline & QPSK & 0 & 97 & $\mathbf{0}$ & 0 & $\mathbf{0}$ & 3 & 0 & 100 & 0 & 0 & $\mathbf{0}$ & $\mathbf{0}$ & 0 & 100 & $\mathbf{0}$ & 0 & 0 & 0 & $\mathbf{0}$ & 100 & 0 & 0 & $\mathbf{0}$ & 0 & 0 & 100 & 0 & 0 & 0 & 0 \\
\hline & 8PSK & 0 & $\mathbf{0}$ & 67 & 0 & 27 & 6 & 0 & 0 & 90 & 0 & 10 & $\mathbf{0}$ & 0 & 0 & 97 & 0 & 3 & 0 & $\mathbf{0}$ & 0 & 100 & 0 & $\mathbf{0}$ & 0 & 0 & 0 & 100 & 0 & 0 & 0 \\
\hline & 4QAM & 0 & $\mathbf{0}$ & $\mathbf{0}$ & 100 & $\mathbf{0}$ & o & 0 & 0 & $\mathbf{0}$ & 100 & $\mathbf{0}$ & $\mathbf{0}$ & 0 & 0 & $\mathbf{0}$ & 100 & $\mathbf{0}$ & $\mathbf{0}$ & $\mathbf{0}$ & 0 & 0 & 100 & $\mathbf{0}$ & 0 & 0 & 0 & 0 & 100 & 0 & 0 \\
\hline & 16QAM & 0 & o & 23 & 0 & 44 & 33 & 0 & 0 & 3 & 0 & 87 & 10 & 0 & 0 & $\mathbf{0}$ & o & 93 & 7 & $\mathbf{0}$ & 0 & 3 & $\mathbf{0}$ & 97 & 0 & 0 & 0 & 0 & 0 & 100 & 0 \\
\hline & 64QAM & o & 3 & 3 & 0 & 34 & 60 & o & 3 & 0 & o & 24 & 73 & o & 0 & 0 & $\mathbf{0}$ & 10 & 90 & 0 & o & 0 & o & 3 & 97 & o & o & o & 0 & o & 100 \\
\hline
\end{tabular}



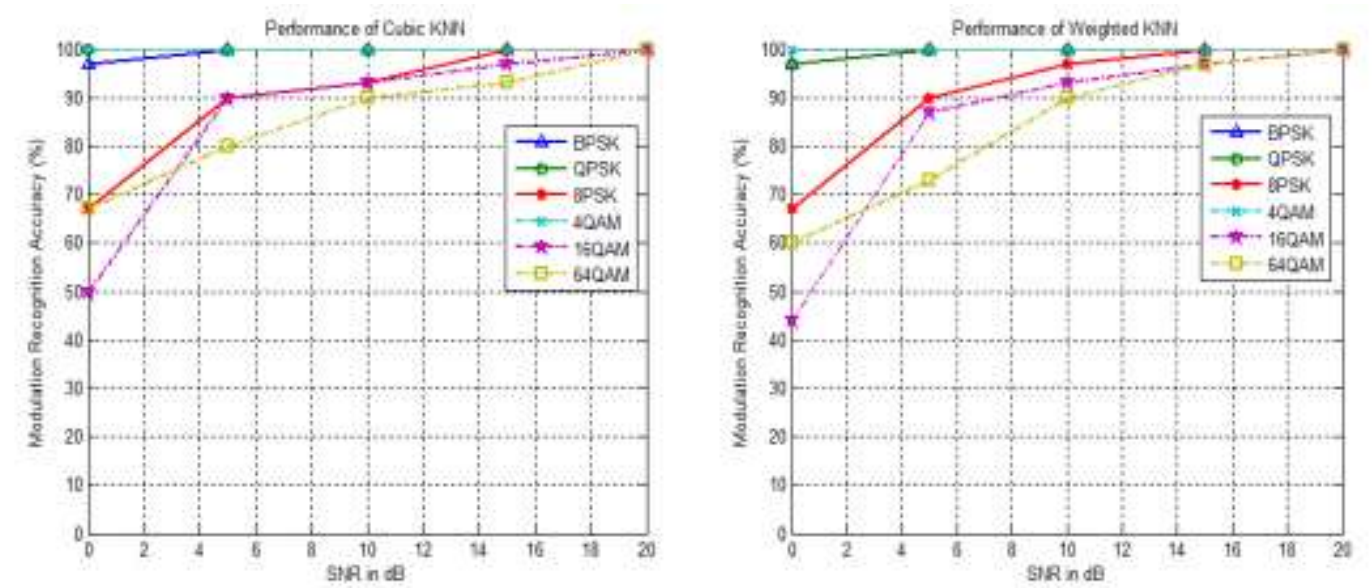

Fig. 5 Performance of Cubic and Weighted KNN Classifiers

The performance comparison of all KNN classifiers is shown in Fig. 6. Among all KNN's Cubic and Medium KNN classifiers achieves better performance in modulation classification i.e. cubic and Euclidean distance functions with $\mathrm{K}$ value of 10 provides the better classification with KNN classifier for AMC of MPSK and MQAM signals.

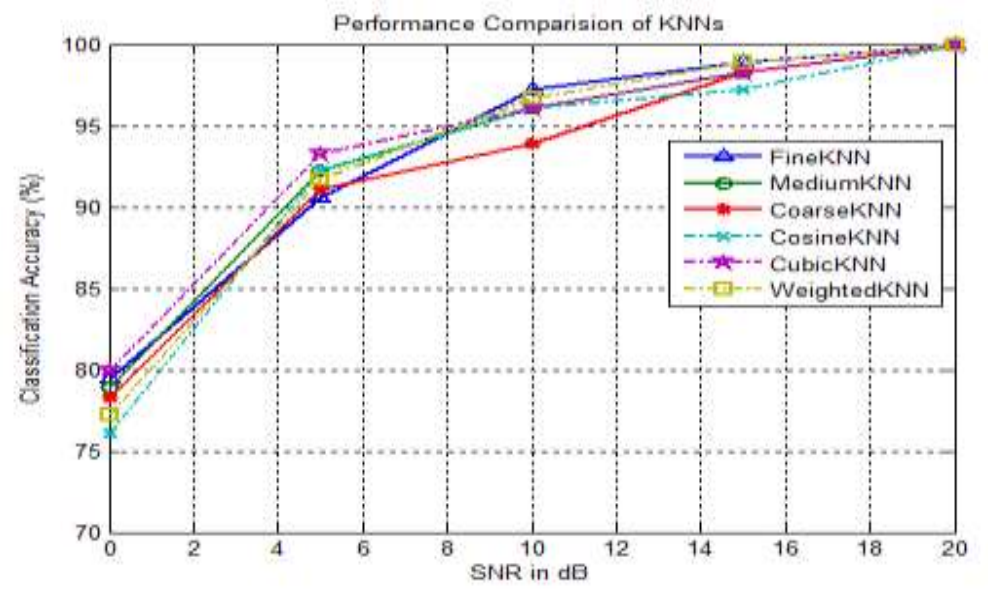

Fig. 6 Performance comparison among KNN Classifiers

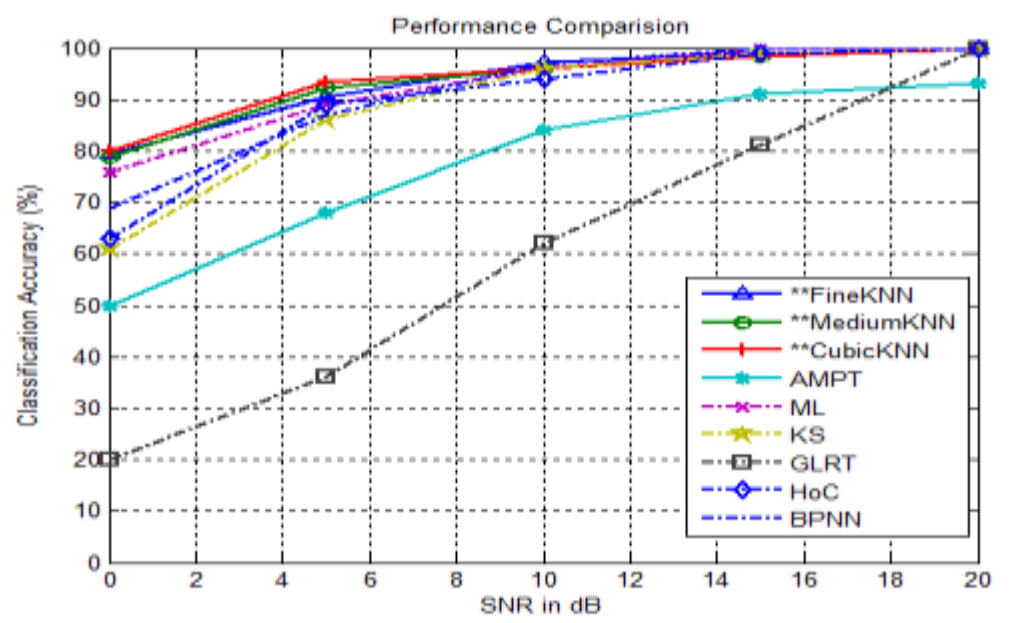

Fig. 7 Comparison of KNN Classifiers with Existing Approaches 
The performance comparison of best three KNN classifiers with existing approaches is shown in Fig. 7. Most of the existing approaches are not considering MPSK, MQAM signals along with MASK and MFSK signals. As the number of modulation techniques increases, then the performances of existing approaches are degraded. With all classes of MPSK and QAM signals, the proposed KNN classifiers achieve better classification accuracy even at lower SNR values than that of existing approaches. From these simulation results, it is proved KNN classifiers provide optimal classification accuracy in terms of modulation recognition under non-ideal channel conditions than the existing approaches.

Table III. Performance of proposed KNN classifiers with different training cases

\begin{tabular}{|c|c|c|c|c|c|c|}
\hline \multirow{2}{*}{ Training $\%$} & \multirow{2}{*}{ Classifier } & \multicolumn{5}{|c|}{ Classification Accuracy (\%) } \\
\hline & & $0 \mathrm{~dB}$ & $5 \mathrm{~dB}$ & $10 \mathrm{~dB}$ & $15 \mathrm{~dB}$ & $20 \mathrm{~dB}$ \\
\hline \multirow{6}{*}{80} & Fine KNN & 76.8 & 92.9 & 98.2 & 99.1 & 100 \\
\hline & Medium KNN & 79.9 & 93.3 & 97.5 & 98.3 & 100 \\
\hline & Coarse KNN & 79 & 93.5 & 97.3 & 98 & 100 \\
\hline & Cosine KNN & 77.4 & 93.2 & 96.8 & 97.8 & 100 \\
\hline & Cubic KNN & 79.8 & 93.3 & 97.4 & 98.4 & 100 \\
\hline & Weighted KNN & 78.3 & 93.8 & 98.3 & 99 & 100 \\
\hline \multirow{6}{*}{70} & Fine KNN & 76.8 & 92.4 & 97.1 & 98.7 & 100 \\
\hline & Medium KNN & 79.7 & 93.7 & 97.6 & 98 & 100 \\
\hline & Coarse KNN & 80 & 92.9 & 96.8 & 97.6 & 100 \\
\hline & Cosine KNN & 78.8 & 93.3 & 96.8 & 97.3 & 100 \\
\hline & Cubic KNN & 79.8 & 93.7 & 97.4 & 97.8 & 100 \\
\hline & Weighted KNN & 78.6 & 93.1 & 98.1 & 98.4 & 100 \\
\hline \multirow{6}{*}{60} & Fine KNN & 76.3 & 92.9 & 97.3 & 98.6 & 100 \\
\hline & Medium KNN & 78.8 & 92.7 & 97.3 & 98.2 & 100 \\
\hline & Coarse KNN & 79.3 & 92.8 & 96.8 & 97.9 & 100 \\
\hline & Cosine KNN & 78 & 92.1 & 96.3 & 97.5 & 100 \\
\hline & Cubic KNN & 78.6 & 92.5 & 97.3 & 98.1 & 100 \\
\hline & Weighted KNN & 80.2 & 94.9 & 99.3 & 99.3 & 100 \\
\hline \multirow{6}{*}{50} & Fine KNN & 75 & 92.8 & 97.2 & 98.6 & 100 \\
\hline & Medium KNN & 78.9 & 92.8 & 97.2 & 98.6 & 100 \\
\hline & Coarse KNN & 78.9 & 92.7 & 96.7 & 98.1 & 100 \\
\hline & Cosine KNN & 78 & 92.8 & 96.3 & 98.1 & 100 \\
\hline & Cubic KNN & 78.9 & 92.8 & 97.1 & 98.5 & 100 \\
\hline & Weighted KNN & 80.3 & 95.6 & 98.7 & 99 & 100 \\
\hline
\end{tabular}

The performance analysis of the proposed KNN classifiers with different percentage of training is shown in Table III. From the results, it is proved that the classification performance of proposed classifiers is better than existing approaches, even with less training.

\section{CONCLUSION}

In this paper, KNN classifiers are developed for AMC of MPSK and MQAM signals. Extraction of different higher order statistical features for each modulation class and selection of appropriate futures for training is presented. The performance of proposed 
KNN classifiers is analyzed under non-ideal channel conditions with various values of SNR. From the simulation results, it is proved that even with more classes of MPSK and MQAM signals, the proposed KNN classifiers achieve more classification accuracy even at lower SNRs than that of existing approaches. Further the research can be extended by incorporating optimization algorithms along with the proposed pattern recognition algorithms, to improve the classification accuracy and to reduce the training time. By establishing a complete communication link with Universal Software Radio Peripherals (USRPs), the realistic signals are traced and the proposed classifiers are applied for the analysis of the performance of proposed algorithms in real time applications.

\section{ACKNOWLEDGMENTS}

This work was supported by Ministry of Electronics \& IT. Authors are very thankful to MeitY-Visvesvaraya PhD Scheme, New Delhi for support to do the research work.

\section{REFERENCES}

[1] Andrea J. Goldsmith, Soon Ghee Chua, "Adaptive Coded Modulation for Fading Channels," IEEE Transactions on Communications, vol. 46, no. 5, pp. 595-602, Mar 1998.

[2] Zhenyu Zhang, Zhong Hua, Yingzhe Liu, "Modulation classification in multipath fading channels using sixth-order cumulants and stacked convolutional auto-encoders," IET Communications, vol. 11, no. 6, pp. 910-915, Nov 2017.

[3] M. Venkata Subbarao, P. Samundiswary, "Automatic Modulation Recognition in Cognitive Radio Receivers using Multi-Order Cumulants and Decision Trees," International Journal of Recent Technology and Engineering, vol. 7, no. 4, pp. 61-69, Nov 2018.

[4] O. A. Dobre, A. Abdi, Y. Bar-Ness and W. Su, "Survey of automatic modulation classification techniques: classical approaches and new trends," IET Communications, vol. 1, no. 2, pp. 137-156, April 2007.

[5] J. A. Sills, "Maximum-likelihood modulation classification for PSK/QAM," Proceedings of IEEE Military Communications Conference, Atlantic City, NJ, USA, vol. 1, pp. 217-220, 1999.

[6] O. A. Dobre, Y. Bar-Ness and Wei Su, "Higher-order cyclic cumulants for high order modulation classification," Proceedings of IEEE Military Communications Conference, Boston, MA, USA, vol. 1, pp. 112-117, 2003.

[7] O. A. Dobre, Y. Bar-Ness and Wei Su, "Robust QAM modulation classification algorithm using cyclic cumulants," Proceedings of IEEE Wireless Communications and Networking Conference, Atlanta, GA, USA, vol. 2, pp. 745-748, 2004.

[8] S. C. Kremer and J. Shiels, "A testbed for automatic modulation recognition using artificial neural networks," Proceedings of Canadian Conference on Electrical and Computer Engineering, Engineering Innovation: Voyage of Discovery, Saint Johns, Newfoundland, Canada vol. 1, pp. 67-70, 1997.

[9] K. L. Davidson, J. R. Goldschneider, L. Cazzanti and J. W. Pitton, "Feature-based modulation classification using circular statistics," Proceedings of IEEE Military Communications Conference, Monterey, CA, USA, vol. 2, pp. 765-771, 2004.

[10] Yun Lin, Xiaochun Xu, Bin Li, Jinfeng Pang and Ruolin Zhou, "The feature extraction and classification for signals based on the S-transform," Proceedings of $2^{\text {nd }}$ International Conference on Measurement, Information and Control, Harbin, pp. 550-553, 2013.

[11] T. R. Kishore, D. S. Sidharth and K. D. Rao, "Analysis of linear and non-linear frequency modulated signals using STFT and hough transform," Proceedings of IEEE International Symposium on Signal Processing and Information Technology (ISSPIT), Abu Dhabi, pp. 490-494, 2015.

[12] M. L. D. Wong and A. K. Nandi, "Blind Phase-Amplitude Modulation Classification with Unknown Phase Offset," Proceedings of $18^{\text {th }}$ International Conference on Pattern Recognition (ICPR'06), Hong Kong, pp. 177-180, 2006.

[13] D. Shimbo, I. Oka and S. Ata, "A Modulation Classification Using Joint Moments with Linear Transform," Proceedings of IEEE Radio and Wireless Symposium, Long Beach, CA, pp. 567-570, 2007.

[14] Chisheng Li, Jing Xiao and Qingyi Xu, "A novel modulation classification for PSK and QAM signals in wireless communication," Proceedings of IET International Conference on Communication Technology and Application (ICCTA 2011), Beijing, pp. 89-92, 2011.

[15] Mohannad Abu-Romoh, Ahmed Aboutaleb, Zouheir Rezki, "Automatic Modulation Classification using Moments and Likelihood Maximization," IEEE Communication Letters, vol. 22, no. 5, pp. 938 941, May 2018.

[16] Jae-Do Jin, Young-Jin Kwak, Kwang-Wook Lee, Kyung Hoon Lee and Sung-Jea Ko, "Modulation type classification method using wavelet transform for adaptive modulator," Proceedings of International 
Symposium on Intelligent Signal Processing and Communication Systems (ISPACS 2004), Seoul, South Korea, pp. 289-292, 2004.

[17] Jian Chen, Yonghong Kuo, Jiandong Li, Fenglin Fu and Yubao Ma, "Digital modulation identification by wavelet analysis," Proceedings of Sixth International Conference on Computational Intelligence and Multimedia Applications (ICCIMA'05), Las Vegas, NV, USA, pp. 29-34, 2005.

[18] O. O. Odejide, C. M. Akujuobi, A. Annamalai and G. Fudge, "Signal and Modulation Type Detection Using Wavelet Transform," Proceedings of WRI World Congress on Computer Science and Information Engineering, Los Angeles, CA, pp. 457-460, 2009.

[19] P. Shih and D. Chang, "An automatic modulation classification technique using high-order statistics for multipath fading channels," Proceedings of 11th International Conference on ITS Telecommunications, St. Petersburg, , pp. 691-695, 2011.

[20] J. L. Barrera, F. E. Hernandez, "Classification of MPSK Signals through Eighth- Order Statistical Signal Processing,” IEEE Latin America Transactions, vol. 15, no. 9, pp. 1601-1607, Sep. 2017.

[21] P. Liu and P. L. Shui, "A new cumulant estimator in multipath fading channels for digital modulation classification," IET Communications, vol. 8, no. 16, pp. 2814-2824, Aug. 2014.

[22] D. C. Chang and P. K. Shih, "Cumulants-based modulation classification technique in multipath fading channels," IET Communications, vol. 9, no. 6, pp. 828-835, Apr. 2015.

[23] Ibrahim Abdel Hafiz El Rube and Nour El-din El-Madany, "Cognitive digital modulation classifier using artificial neural networks for NGNs," Proceedings of Seventh International Conference on Wireless and Optical Communications Networks - (WOCN), Colombo, pp. 1-5, 2010.

[24] Z. Wu, S. Zhou, Z. Yin, B. Ma and Z. Yang, "Robust Automatic Modulation Classification Under Varying Noise Conditions," IEEE Access, vol. 5, pp. 19733-19741, 2017.

[25] M. W. Aslam, Z. Zhu and A. K. Nandi, "Automatic Modulation Classification Using Combination of Genetic Programming and KNN," IEEE Transactions on Wireless Communications, vol. 11, no. 8, pp. 2742-2750, August 2012.

[26] Lubing Han, Zan Li, O A Dobre, "Low complexity automatic modulation classification based on orderstatistics," IEEE Transactions on Wireless Communications, vol. 16, no. 1, pp. 400-411, Jan. 2017.

[27] M. W. Aslam, Z. Zhu and A. K. Nandi, "Automatic Modulation Classification Using Combination of Genetic Programming and KNN," IEEE Transactions on Wireless Communications, vol. 11, no. 8, pp. 2742-2750, 2012.

[28] Lubing Han, Zan Li, O A Dobre, "Low complexity automatic modulation classification based on orderstatistics," IEEE Transactions on Wireless Communications, vol. 16, no. 1, pp. 400-411, 2017.

[29] Vitola J, Poz, F, Tibaduiza D.A., Anaya M, "A Sensor Data Fusion System Based on k-Nearest Neighbor Pattern Classification for Structural Health Monitoring Applications," Sensors, vol. 17, no. 2, pp. 417-426, 2017. 